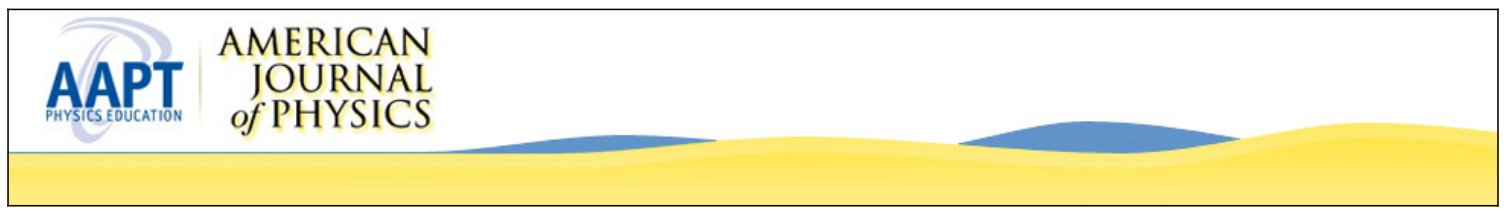

\title{
Microwave propagation in dielectric fluids
}

W. P. Lonc

Citation: American Journal of Physics 48, 648 (1980); doi: 10.1119/1.12043

View online: http://dx.doi.org/10.1119/1.12043

View Table of Contents: http://scitation.aip.org/content/aapt/journal/ajp/48/8?ver=pdfcov

Published by the American Association of Physics Teachers

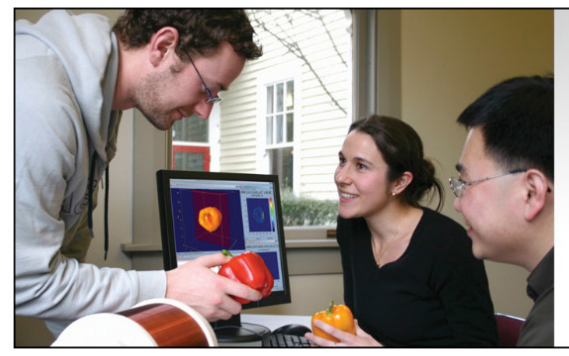

\section{Teach NMR and MRI}

Hands-on education with Terranova-MRI Includes complete student guide with 12 experiments and online videos. 


\title{
Microwave propagation in dielectric fluids
}

\author{
W. P. Lonc, S.J. \\ Physics Department, Saint Mary's University, Halifax, Canada B3H 3 C3 \\ (Received 30 October 1979; accepted 12 December 1979)
}

An undergraduate experiment designed to verify quantitatively the effect of a dielectric fluid's dielectric constant on the observed wavelength of microwave radiation propagating through the fluid is described. The fluid is castor oil, and the results agree with the expected behavior within 5\%, assuming a dielectric constant of $2.65 \pm 0.05$ for castor oil at $3-\mathrm{cm}$ wavelength.

\section{INTRODUCTION}

An initial study at the undergraduate level of the relationship between the wavelength of an electromagnetic wave traveling in an unbounded polarizable (dielectric) medium and the macroscopic dielectric constant of the medium may be carried out at microwave frequencies using rather simple equipment. ${ }^{1}$ On the basis that the wavelength can be measured using a "standing-wave" technique, it remains to devise a suitable experimental procedure for measuring the wavelength of standing waves in a polarizable medium. In particular, the procedure is comparatively simple if the study is performed on a dielectric fluid.

The objective is to make available to readers of this Journal a tested experiment which could be used as a laboratory exercise. This exercise, no more than three hours long, will verify the shortening of wavelength in castor oil at room temperature to within $5 \%$ of the expected value, assuming a value of $2.65 \pm 0.05$ for the dielectric constant of castor oil at a nominal wavelength of $3 \mathrm{~cm} .{ }^{2}$ It is assumed that for the purposes of this experiment, castor oil is sufficiently independent of geographical factors. Hence in some applications, such as an undergraduate experiment, this simple method would suffice, rather than the more precise techniques which require relatively more complicated apparatus, as well as inclusion of effects on the propagation arising from any departure from an ideally unbounded medium.

\section{THEORY}

For electromagnetic waves of frequency $f$ and wavelength $\lambda$, traveling in an unbounded medium characterized by a permittivity $\epsilon$ and permeability $\mu$, it may be shown that these quantities are related through the equation

$$
\lambda=1 /(f \sqrt{\mu \epsilon}) \text {. }
$$

Assuming that the permeability of the medium is numerically equal to that of air, and assuming that the frequency $f$ remains constant, then the wavelengths $\lambda$ and $\lambda_{0}$ are related to the dielectric constant $K$ by

$$
\lambda=\lambda_{0} / \sqrt{K},
$$

where $\lambda_{0}$ is the free-space wavelength. Hence, a measurement of the wavelengths, combined with a known value for $K$, enable one to test the theory expressed in Eq. (2).

\section{METHOD}

Assuming a standing-wave method, the response of a detector will depend on its location within the standing-wave pattern, which exists between the reflector and the detector. If the detector could be moved relative to this pattern, then its response as a function of position would be an indication of the standing-wave pattern, and hence the wavelength could be obtained from this information. If, however, motion of the detector is not convenient, especially if the distance could not be measured conveniently or if the detector cannot be operated in a liquid, then an alternative procedure would be to move the reflector relative to the detector: this is the procedure used in this experiment. In our experiment, the reflector consists of a metal plate attached to the carriage of a traveling microscope (the telescope is temporarily removed from the instrument), allowing a measurement of relative position to a fraction of a millimeter. Any device enabling linear motion measurement to $1 \mathrm{~mm}$ or so should suffice.

\section{EXPERIMENTAL PROCEDURE}

On the basis of the theory, a medium characterized by a dielectric constant of approximately 3 (such as castor oil) should show a decrease in wavelength by a factor of approximately 1.7 , from its free-space value $\lambda_{0}$, which should be easily observable with undergraduate-level microwave equipment. It only remains to ensure that the necessary

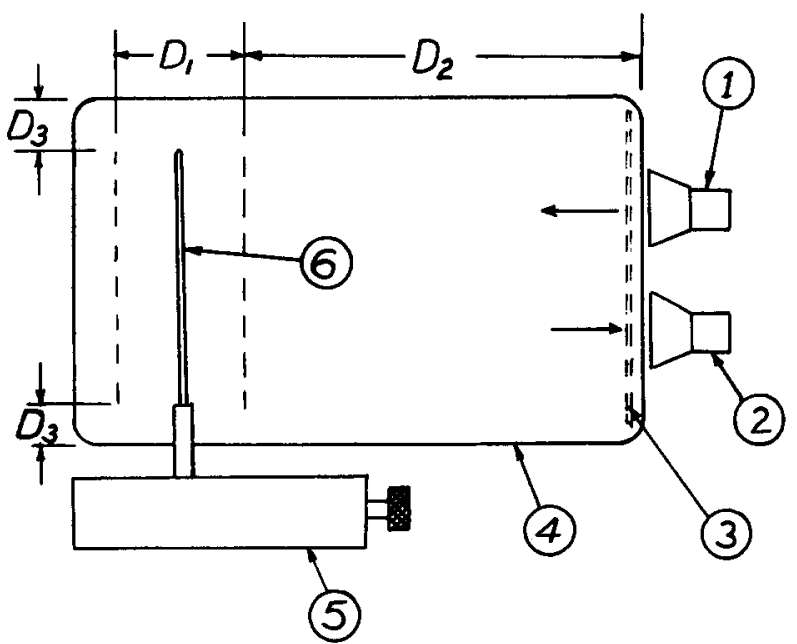

Fig. 1. Top view of general layout of the apparatus. 1: transmitter and associated electronics; 2 : receiver horn and associated electronics; 3 : metal screen extending upwards from the top of the container; 4 : plastic container; 5 : calibrated linear motion assembly; 6 : metal plate for reflecting the microwaves; $D_{1}$ : distance over which the reflecting surface travels; $D_{2}$ : minimum distance between the reflecting surface and the front of the container; $D_{3}$ : distance between the sides of the container and the reflecting surface. 

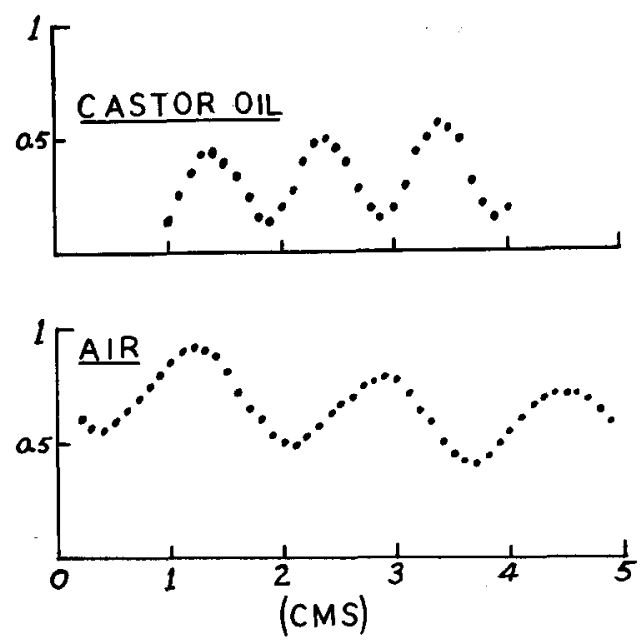

Fig. 2. Standing-wave patterns for castor oil (upper trace) and air (lower trace). The horizontal axis represents the position of the reflecting surface, measured in centimeters from an arbitrary reference point. The vertical axis represents the received amplitude, in arbitrary units.

conditions, such as unbounded propagation, are sufficiently well met in the experimental procedure.

In Fig. 1 the waves are reflected from the moveable reflector and thus produce a standing wave in front of this surface. Evidently there will be reflection of the radiation, not only from the reflector, but also from the various surfaces associated with the container and carriage assembly. However, once the standing-wave pattern has been plotted, as in Fig. 2, it will be clear where the relevant regions of the pattern are located. In other words, spurious reflections will occur in a manner such that they can be easily distinguished from the desired pattern.

Aside from the microwave equipment, the only additional apparatus is: (i) a plastic container of the type used for food storage in a refrigerator, approximately $30 \times 15 \times 15 \mathrm{~cm}$ and (ii) a movea ble flat reflector approximately $12 \times 7 \mathrm{~cm}$. These dimensions are not critical: the container need only be large enough to allow the moveable reflector to move freely over a distance $D_{1}$ of 2 or 3 wavelengths within the medium, and to allow a minimum distance $D_{2}$ of several wavelengths between the reflector and the container's front surface.

The moveable reflector was attached to a calibrated carriage, such as a traveling microscope, and fitted in such a way that it was completely immersed in the liquid. In our experiment, the nearest distance $D_{3}$ between any point of the reflector and the sides of the plastic container was no less than $3.5 \mathrm{~cm}$, which corresponds to approximately two wavelengths in the medium.

It is recommended that a metallic screen be placed at the front of the container in such a way that it reaches from the surface of the liquid upwards, thereby shielding the reflector support structure from incident radiation. Hence, the only moving surface receiving a significant amount of incident radiation is the reflector. Neglect of this particular precaution can lead to quite serious spurious reflections, which can then dominate the standing-wave pattern, leading to an erroneous value for the wavelength.

The first step in the experiment could be to verify the relationship expressed in Eq. (1) for propogation in air, which would mean that the observed wavelength would be the value consistent with a dielectric constant equal to unity; this step presupposes an independent measurement of the frequency. If an independent measurement of the frequency is not available, however, then Eq. (1) as such is not verified, but rather Eq. (2) is now appropriate, implying that the measured value of $\lambda$ in air will be used as a reference value, relative to which the value of $\lambda$ for propagation in the dielectric liquid is expected to be smaller.

In this first step, therefore, all the elements of the apparatus, including the plastic container, are in place, as in Fig. 1. The only difference between this configuration and the one when propogation through the dielectric liquid is being studied, is that in the first instance, the plastic container contains only air, whereas in the second, the container contains the dielectric liquid.

The next step in the experiment is to pour the dielectric liquid into the plastic container and immerse the reflector in the liquid. Except for the presence of the dielectric liquid, the experimental arrangement is exactly the same as for Sec. I; hence, any changes in the standing-wave pattern must be due to the presence of the liquid.

\section{RESULTS}

Inspection of the standing-wave patterns in Fig. 2 indicates that the data is sufficiently well-defined to warrant acceptance as valid, and hence the calculated value for $\lambda$ in castor oil should be valid. On the basis of the pattern in Fig. 2, the wavelength $\lambda$ of the radiation in the castor oil is found to be $2.0 \mathrm{~cm}$, which is to be compared with the expected value of $1.98 \mathrm{~cm}$ if the dielectric constant for castor oil at this frequency is taken to be 2.65 .

\section{CONCLUSION}

In conclusion, the shortening of the wavelength of microwave radiation propogating in a dielectric medium (such as castor oil) as predicted from classical electromagnetic theory can be observed with relative ease using simple $3-\mathrm{cm}$ equipment, in the course of a typical undergraduate laboratory period. In addition, the correlation between the observed dielectric constant, as calculated from the theory, and that of accepted values is within the limits of typical experimental uncertainty.

${ }^{1}$ A method employing a slotted line procedure is described by E. F. Carr and G. S. Harmon in Am. J. Phys. 47, 281 (1979).

${ }^{2} \mathrm{~A}$ value of 2.68 is given in the American Institute of Physics Handbook [3rd ed., edited by Dwight E. Gray (McGraw-Hill, New York, 1972), p. 5-137] for the dielectric constant of castor oil at $3 \mathrm{GHz}$. On the basis that the value would decrease by no more than a few percent for a three-fold increase in frequency (no resonances are known in this part of the spectrum for castor oil), a value of 2.65 with an uncertainty of at least 0.05 appears to be justifiable. 